Article

\title{
Experimental Study of the Lubrication Mechanism of Micro-Spherical Solid Particles between Flat Surfaces
}

\begin{abstract}
Abdullah A. Alazemi
Department of Mechanical Engineering, College of Engineering and Petroleum, Kuwait University, P.O. Box 5969, Safat 13060, Kuwait; a.alazemi@ku.edu.kw

Abstract: In this study, a novel apparatus was designed and constructed to perform micro-sliding friction experiments while simultaneously observing the motion of micro-sized spheres using a visual inspection technique. The apparatus comprises a precision elevation stage that is used to elevate a flat mica disk, with the microspheres on top of it, to bring it into contact with a stationary surface and apply low loads on the contact. During micro-sliding experiments, it was found that the velocity of the center of the microsphere was half the velocity of the mica disk; in addition, friction force measurements revealed a very low coefficient of friction (about 0.03), indicating the rolling motion of the microspheres. The main outcome of our study was the verification of the hypothesis that spherical particles can be used to avoid direct contact among flat surfaces and can also introduce rolling motion within the system. The pure rolling motion of the microspheres sandwiched between a stationary and a moving flat surface supports the idea of adding rigid spherical particles to oil lubricants to further reduce friction and wear in the system by performing as micro and nanoscale ball bearings.
\end{abstract}

Publisher's Note: MDPI stays neutral with regard to jurisdictional claims in published maps and institutional affiliations.

Keywords: microspheres; lubrication mechanism; friction; rolling motion; in situ

\section{Introduction}

Most mechanical systems involve energy losses due to the presence of friction and wear between moving surfaces. Commonly, liquid lubricants are used to lower wear and frictional losses by minimizing direct surface contact. Nevertheless, high frictional force and wear loss arises in boundary and mixed lubrication regions since fluid lubricant film thickness is less than $1 \mu \mathrm{m}$ [1]. In boundary and mixed lubrication regions, asperities interaction happens even with the existence of a liquid lubricant and, as a result, high wear and friction occur. Therefore, numerous research studies have been dedicated to advance the tribological properties of existing liquid lubricants.

Recent research reports have pointed to the promising tribological results of nanoparticles when combined with 2D materials such as graphene. It has been shown that metal nanoparticle/graphene nanocomposites can be utilized as superior lubricant additive materials as well as solid lubricants or as film lubrication because of their exceptional self-lubricating properties [2]. Shi and Jiang [3] indicated that hybrid graphene and copper nanostructures can enhance the tribological performance when added to solid lubricant coatings. Furthermore, it has been shown that the combination of metal nanoparticles with two-dimensional nanosheets is an efficient method to improve the tribological properties of polymers [4].

Several studies have demonstrated that adding rigid nanoparticles to a liquid lubricant can result in decreasing friction force and wear damage in the boundary and mixed lubrication regions [5-8]. Previous experimental works have demonstrated that various forms of solid particles, of sizes ranging from 1 up to 50,000 nm, could be employed as lubricant additives for better tribological performance [9-11]. However, there is a lack of complete knowledge of the lubrication technique of these solid nanoparticles [12]. Various 
lubrication techniques using these solid nanoparticles have been theorized as they might be rolling between moving surfaces, or they can form a shielding film on contacting surfaces [13]. The study of the lubrication technique of solid nanoparticles can be vital to predict their physical stability and to enhance their role as anti-friction and anti-wear oil additives.

Experimental investigations of the lubrication mechanism of solid nanoparticles were performed post-tribotests using X-ray photoelectron spectroscopy (XPS) [14], scanning electron microscopy (SEM) [15], and transmission electron microscopy (TEM) [16]. These experimental studies on the wear scars of sliding surfaces suggested that the lubrication mechanism of nanoparticles could be due to (a) the development of a tribo-film within the area of interaction $[5,15],(b)$ rolling motion of nanoparticles acting as nanoscale ball bearings [17-19], and (c) third-body solid particles preventing direct contact between sliding surfaces [20,21]. However, the proposed lubrication mechanisms are hypothetical, and no clear proof was provided.

Moreover, the rapid increase in energy demand due to the fast population growth combined with the demand to reduce pollution emissions necessitate the improvement in the tribological performance of mechanical systems and reducing their energy consumptions. Several studies [22-25] have demonstrated the viable role of biodiesel products to enhance the tribological performance of fossil fuels without the need to modify the diesel engine. Furthermore, recent research studies [26,27] suggested that the addition of nanoparticles to the biodiesel blends could further advance both engine performance as well as emission characteristics. Mujtaba et al. [26] attributed the improved tribological performance of nanoparticles and biodiesel blends to the presence of a lubricating film between the metallic contacts created by the nanoparticles. Consequently, different lubrication mechanisms coexist such as ball-bearing mechanism, protecting film formation, polishing, and mending effects. However, the ball-bearing mechanism is theorized, and no direct proof was offered. The major purpose of our work is to verify the hypothesis that micro- and nano-spherical particles could introduce rolling motion within contacting surfaces and, as a result, reduce friction and wear. Therefore, the focus of the current study is on examining the lubrication mechanism of spherical solid particles without liquid lubricants to verify and visualize their motion between mating surfaces.

Various factors can affect the lubrication interface such as operating conditions, lubricant viscosity, surface roughness, etc. Nikolakopoulos et al. [28] indicated that even the aging of engine lubricant results in a different piston ring tribological performance due to the change in lubricant viscosity. To minimize wear and avoid the breakdown of mechanical components, numerous researches [29-33] have suggested changing the surface morphology by artificially creating microscopic features on the surface of sliding pairs. Thus, using an appropriate surface roughness will advance the lubrication performance and minimize the friction and wear in the system [30].

Numerical models have been developed to investigate the effect of clearance ratio, speed, and lubricant type on the tribological performance of journal bearings [31-33]. These models can be useful tools to study the bearing lubrication challenges and to predict the performance of mechanical systems in the boundary and transient lubrication regions. Ghaednia and Jackson [12] developed a model to investigate the significance of nanoand micro-sized particles in contact with rough surfaces on the tribological performance. However, the obtained results were inconclusive as to whether the solid nanoparticles would prompt abrasive wear or create tribolayers on sliding surfaces. They suggested that a carefully designed experiment could be useful to study and verify the lubrication mechanism of solid particles.

In the last decade, numerical studies using molecular dynamics (MD) have performed to explore the friction force and wear phenomena at the nanoscale $[13,34,35]$. The obtained results illustrated that the variation in the shape and type of nanoparticles would result in different lubrication mechanisms [13]. Additionally, experimental studies have demonstrated that the tribological performance of solid nanoparticles is more vital in the 
boundary and mixed lubrication regions compared to the hydrodynamic region $[17,18]$. However, as a result of the complexity of physical and chemical reactions in the friction process, there has not been enough conclusive evidence to explain the lubrication mechanisms of micro- and nano-sized particles [36]. Therefore, an experimental investigation of the motion of nano- and micro-sized solid particles can help identify the lubrication mechanism of these particles when added to liquid lubricants.

In the present work, the lubrication mechanism of micro-sized solid spherical particles placed between two flat surfaces was experimentally investigated. An optical micro-sliding instrument was built to perform a micro-sliding movement of one of the flat surfaces while in situ to observe the motion of the micro-particles. This instrument comprises a precision elevation stage to lift one of the flat surfaces, with microspheres on top of it, until it comes in contact with the upper stationary surface. Afterward, a piezoelectric actuator was utilized to move the lower flat surface with the microspheres against the fixed upper flat surface at a low speed. Concurrently, the motion of the micro-sized spheres during the linear movement of the flat surface was observed using a visual inspection technique. The obtained results proved the pure rolling motion of the microspheres in the middle of stationary and moving surfaces. This result supports that micron-sized spherical particles can be used as ball bearings at the microscale to further reduce friction and wear, and advance the tribological performance of liquid lubricants.

\section{Experimental Section}

\subsection{Description of the Optical Micro-Sliding Instrument}

In the current study, an optical micro-sliding (OMS) instrument was utilized to experimentally examine the movement of micro-sized spheres placed in between two flat disks. The OMS apparatus is a modified version of the optical micro-apparatus used for adhesion and friction measurements in a previous investigation [37]. The modification involved substituting the steel sphere positioned at the free end of a cantilever beam arrangement by a flat mica disk. The mica disk was selected due to its atomically smooth surface (arithmetic mean $\left(R_{a}\right)$ surface roughness under $10 \mathrm{~nm}$ ).

The schematic and photograph of the OMS instrument are shown in Figure 1. The OMS apparatus comprises a high-precision elevation stage for the vertical drive of the mica disk. The high accuracy elevation stage (ES-100, by Physik Instrumente, Karlsruhe, Germany) has a height travel range from 0 to $26 \mathrm{~mm}$ with a $0.2 \mu \mathrm{m}$ resolution. A micro-step control system (SMC Pollux, by Physik Instrumente, Karlsruhe, Germany) was used to control the vertical movement of the elevation stage. Furthermore, the OMS apparatus includes a piezoelectric actuator motor for horizontal movement of the mica disk. The piezoelectric actuator ( $\mathrm{P}-$ 216.90, by Physik Instrumente, Karlsruhe, Germany) had a 0-180 $\mu$ m horizontal movement range and resolution of $1.8 \mathrm{~nm}$. The control of the displacement of the piezoelectric actuator was accomplished via a digital piezo controller (E-517, by Physik Instrumente, Karlsruhe, Germany). A miniature load cell (FUTEK model LSB200, CA, USA) with a $0.2 \mathrm{~N}$ capacity was utilized to measure the friction force during the micro-sliding tests. In this investigation, micro-sized stainless-steel spheres with a diameter less than $50 \mu \mathrm{m}$ (supplied by Cospheric LLC, Goleta, CA, USA) were used to explore their motion between two flat disks (see Figure 2). The micro-sized steel spheres were distributed on the top of the mica disk, and then carried into contact with the upper stationary transparent window by the elevation stage. After that, the piezoelectric motor was operated to move the mica disk, which was below the steel microspheres, against the fixed sapphire disk at a very slow sliding speed. In situ observation of the contact interface was conducted using an optical microscope placed over the upper transparent window and attached to a digital camera (see Figure 1). The OMS apparatus comprises a fiber-optic displacement sensor (from Philtec, Inc., Annapolis, MD, USA) to accurately quantify the vertical travel distance of the free end of the cantilever beam (at the location of the flat mica disk). This high-precision fiber-optic displacement sensor had a resolution of $40 \mathrm{~nm}$. 


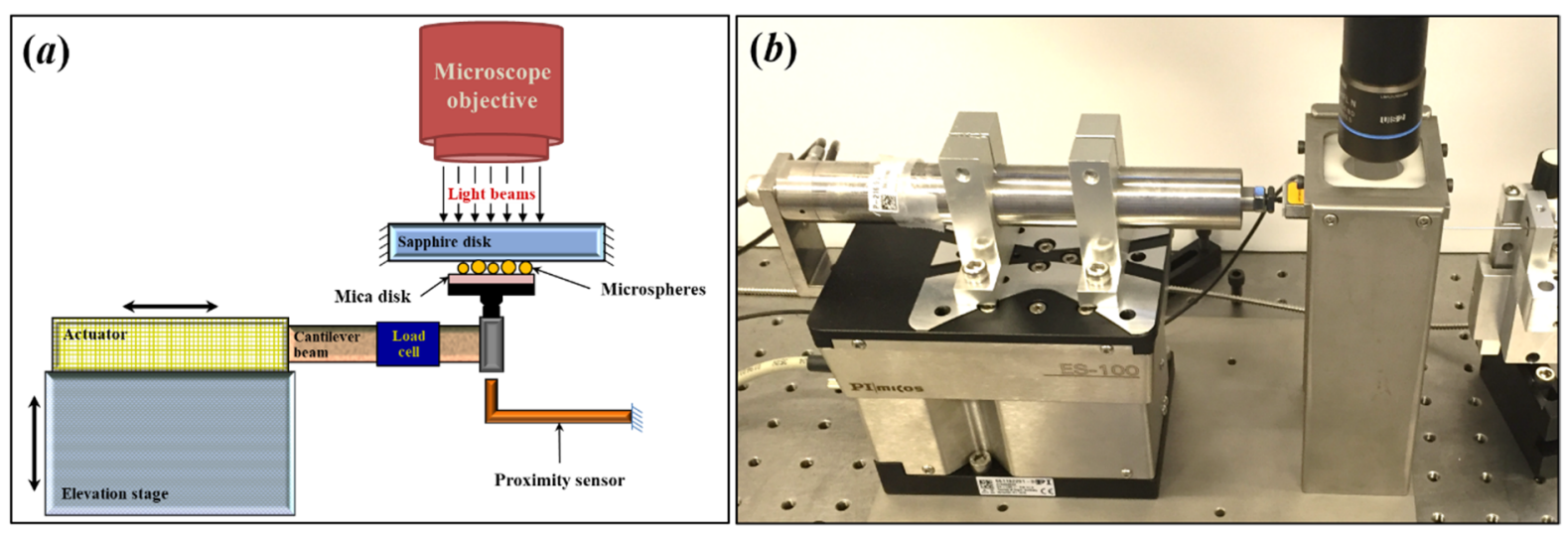

Figure 1. (a) Schematic and (b) photograph of the optical micro-sliding instrument.
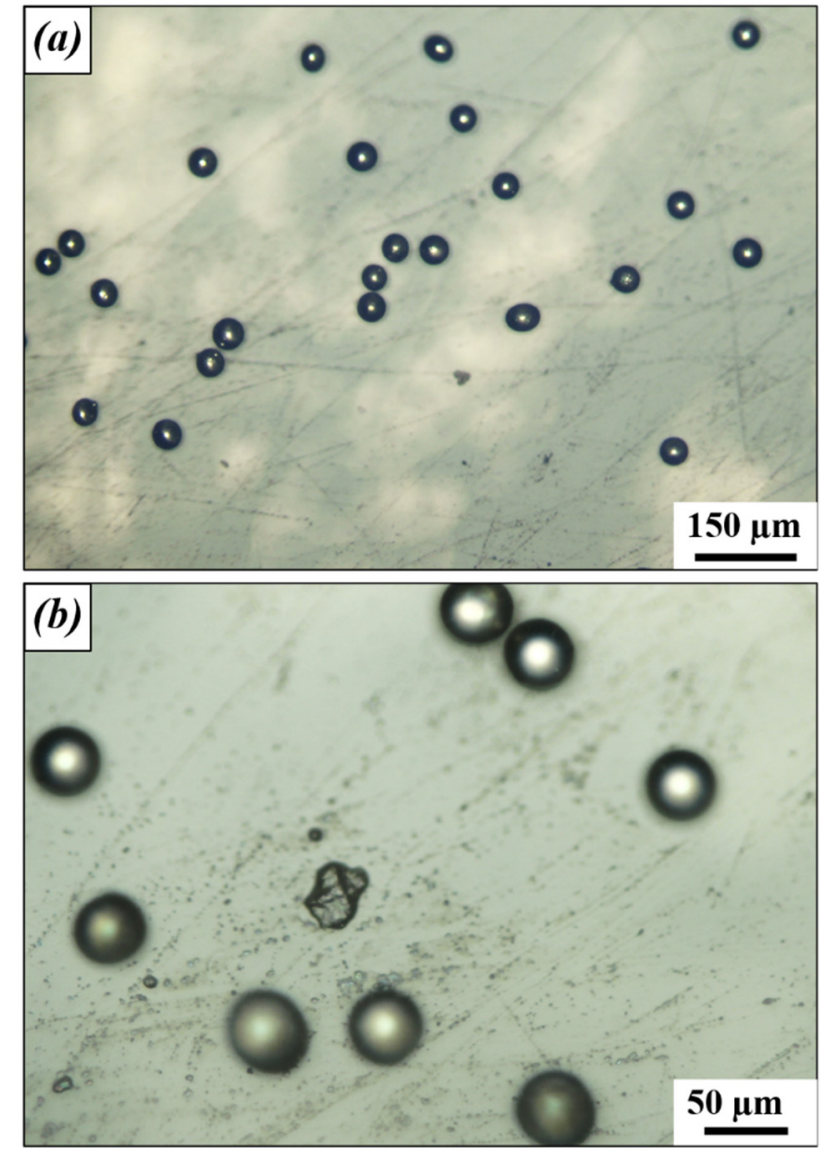

Figure 2. Optical micrographs using (a) $20 \times$ and (b) $50 \times$ magnification lens of the micro-sized stainless-steel particles.

The morphology of the stainless-steel particles was examined via a scanning electron microscope, JEOL JSM-7001F, Japan, operating at an acceleration voltage of $20 \mathrm{kV}$. Furthermore, chemical analysis of stainless-steel particles was studied using EDS. To perform this study, a powder of stainless-steel particles was scattered on copper tape (on an SEM sample holder). Figure 3 depicts SEM micrographs at different magnifications of the stainless-steel particles. The SEM micrographs revealed the spherical structure of stainless-steel particles with a diameter ranging from $41 \mu \mathrm{m}$ to $48 \mu \mathrm{m}$. Because of the surface roughness of the mating surfaces, the solid particles can occupy the gap formed by the asperities of mating surfaces to enlarge the real area of contact and, therefore, lower the surface contact stresses. As a result of the reduction in contact stresses, the wear rate within the system is expected to drastically diminish. Additionally, the spherical structure (with minor shape deviation) 
of the stainless-steel particles can be favorable when used in mechanical systems in reducing friction and wear losses by operating as a ball bearing on the micro-scale. Moreover, as shown in Figure 3c, the stainless-steel particles demonstrated nanometer scale surface irregularities (less than $50 \mathrm{~nm}$ ) that confirmed their smooth surface. The chemical analysis using EDS of stainless-steel particles is shown in Figure 4. The results of the EDS analysis, presented in Figure $4 \mathrm{~b}$, indicate that the particles had an elemental composition of $69.0 \%$ iron, $18.1 \%$ chromium, and $8.6 \%$ nickel, in addition to a small content of other elements, which are practically similar to the stainless-steel alloy material [38].

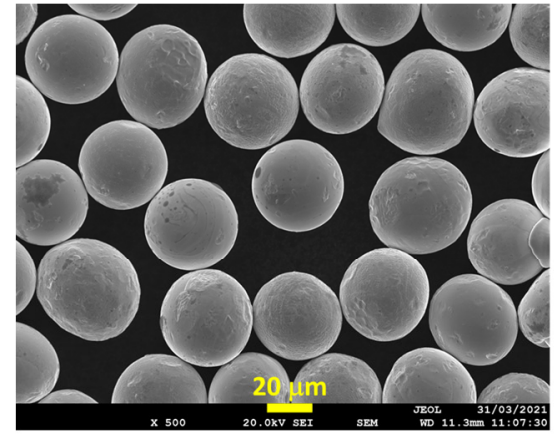

(a)

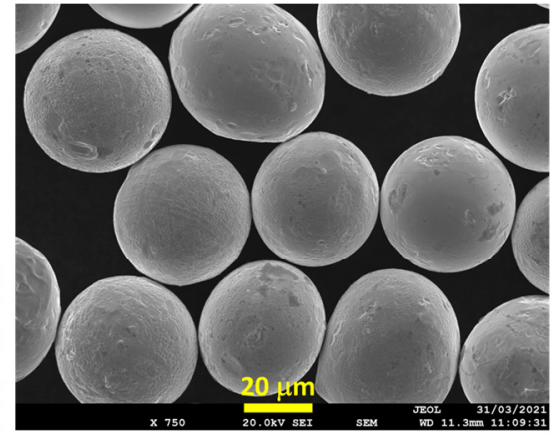

(b)

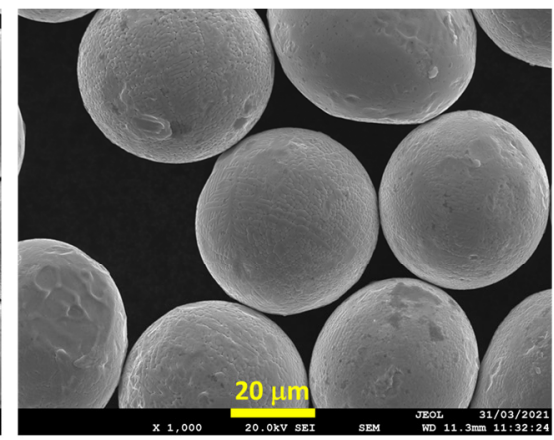

(c)

Figure 3. Scanning electron microscopy (SEM) micrographs of stainless-steel particles at different magnifications: (a) $\times 500$; (b) $\times 750$; and (c) $\times 1000$.

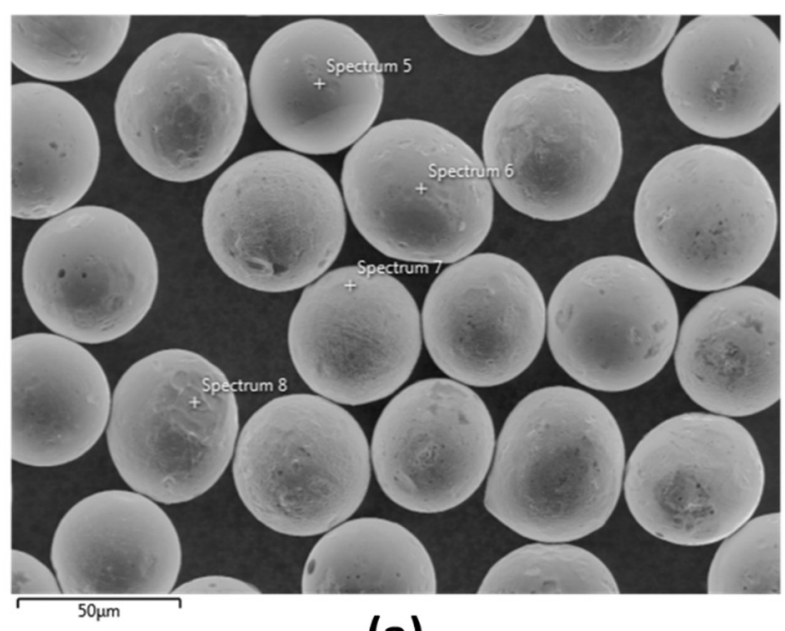

(a)

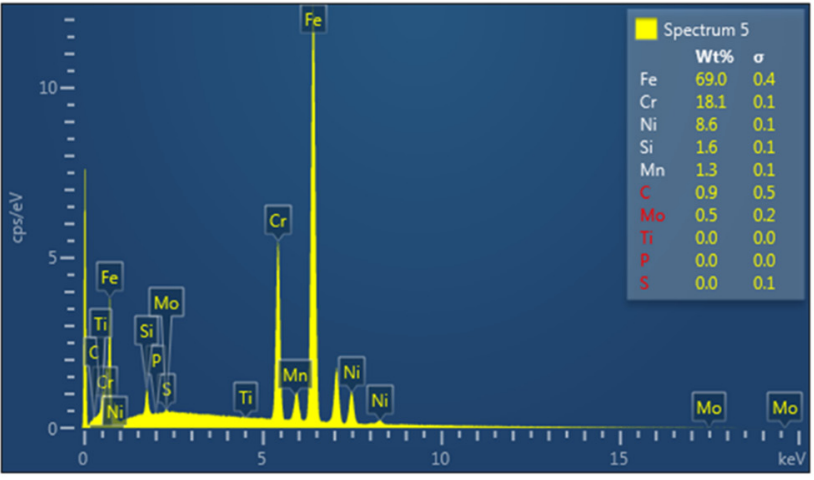

(b)

Figure 4. (a) SEM micrograph and (b) energy dispersive X-ray spectrum of stainless-steel particles.

\subsection{Analysis of Contact Area and Image Processing}

Initially, the mica disk that carries steel microspheres was moved upward via the elevation stage. As soon as the stainless-steel microspheres touched the upper stationary disk, a contact zone was produced in the midpoint of each microsphere. The area of contact between the microspheres and the upper disk can be observed in situ via the microscopecamera system. For a non-conformal contact, Newton's rings interference pattern was produced around the contact area of each microsphere, and this pattern can be used to detect the center of the area of contact (see Figure 5). Furthermore, the dark area in the middle of the contact, as shown in Figure 5, which represents the contact area, is the zeroth-order destructive interference fringe [39]. 

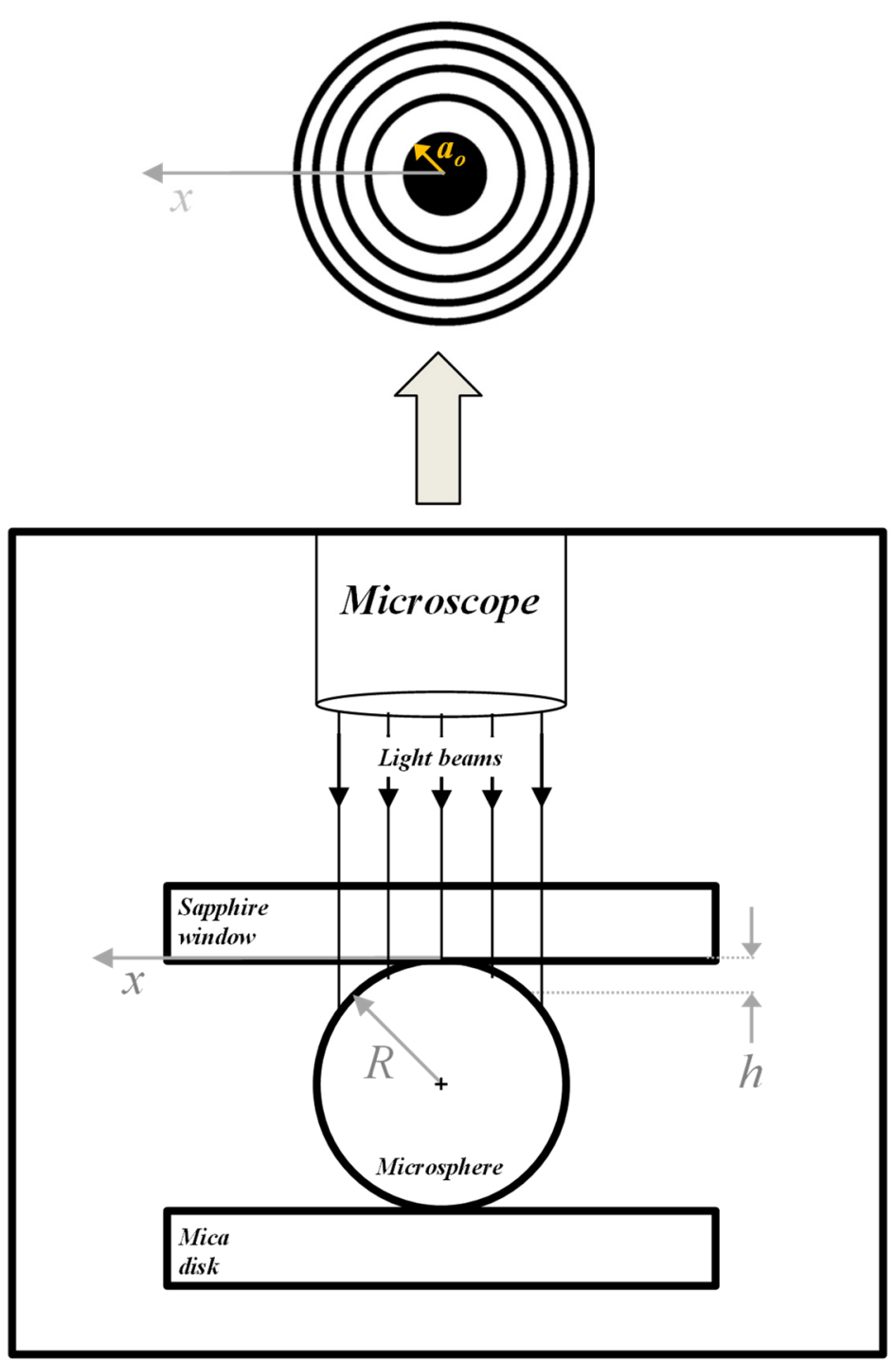

Figure 5. Schematic of contact configuration with an example (top) of Newton's rings interference pattern.

Next, the flat mica disk, carrying the microspheres, was moved horizontally below the fixed transparent window using the piezoelectric actuator. Visual inspection of the contact interface was conducted using the optical microscope standing over the upper transparent window and connected to a camera. Furthermore, the video of the interacting surfaces throughout a micro-sliding cycle was used to trace the center of a microsphere and, thus obtain the relative horizontal movement of the microsphere. Video recording of the contact interface was performed at a rate of 30 frames/s using the digital camera (with a resolution of 18 megapixels). In post-test processing, the acquired video was used to calculate the relative horizontal displacement through the variation in the $x-y$ coordinates of the midpoint of the examined microsphere. This method permits precise measurement of the horizontal movement of the microsphere throughout the linear microsliding experiment. To improve the image resolution and sharpness of acquired videos, a high-magnification lens $(20 \times$ or $50 \times)$ in combination with the highest pixels in the camera (18 megapixels) was utilized. Consequently, the optical microscope used in this study was able to achieve an image resolution of up to $0.55 \mu \mathrm{m}$.

\subsection{Experimental Procedure}

In this study, the tested sapphire specimen was cleaned using acetone and wiped before each test. A freshly cleaved mica disk that had a diameter of $12 \mathrm{~mm}$ and a thickness 
of $0.21 \mathrm{~mm}$ was used as the lower flat surface. As shown in Figure 1a, the mica disk was positioned on the free end of the cantilever beam setup. Stainless-steel microspheres were distributed randomly on the top of the mica disk. A sapphire window $27 \mathrm{~mm}$ in diameter and $1 \mathrm{~mm}$ in thickness was utilized as the upper flat surface. The sapphire window had a modulus of elasticity of $400 \mathrm{GPa}$ and Poisson's ratio of 0.29 . In addition, the transparency of the sapphire window permitted the visualization of the microspheres beneath it.

In this study, the precision elevation stage was used to elevate the flat mica disk, with the microspheres on top of it, to bring it into contact with the stationary sapphire surface. The slow vertical movement (equal to $0.1 \mu \mathrm{m} / \mathrm{s}$ ) was executed via the elevation stage until surface contact initiation occurs. A proximity sensor located below the mica disk was employed to measure its vertical displacement and the applied normal load as it moves toward the upper sapphire window. Consequently, when surface contact is initiated, the proximity sensor will read no movement of the mica disk, and then the vertical input displacement by the elevation stage was stopped. Next, visual inspection using the optical microscope with the digital camera was used to locate a microsphere in contact with the upper sapphire window. Afterward, a micro-sliding test was performed by moving the mica disk horizontally and concurrently observing the motion of the microsphere. In micro-sliding experiments, the piezoelectric actuator was operated to reciprocate the flat mica disk with the microspheres against the fixed sapphire window at a slow speed. Micro-sliding experiments were performed at a low operational frequency $(0.08 \mathrm{~Hz})$ in order to carefully examine the motion of micro steel particles between two flat bodies. During the micro-sliding tests, the input displacement function given to the piezoelectric actuator to move the mica disk was a sinusoidal cycle with an amplitude of $70 \mu \mathrm{m}$ (see Figure 6). At the same time, the optical microscope, in conjunction with the digital camera, was used to video record the motion of the microsphere. Subsequently, the horizontal output movement of the microsphere in the micro-sliding experiment was obtained by processing the recorded video. In this investigation, the data collection and the control of the piezoelectric motor elevation stage were accomplished via LabView software and using a $20 \mathrm{kHz}$ sampling rate. In this investigation, all experiments were performed at room temperature, and all tests were conducted several times to confirm the repeatability and reliability of the obtained results. The measured sliding velocities and friction force values were obtained with a maximum error of approximately $7 \%$.

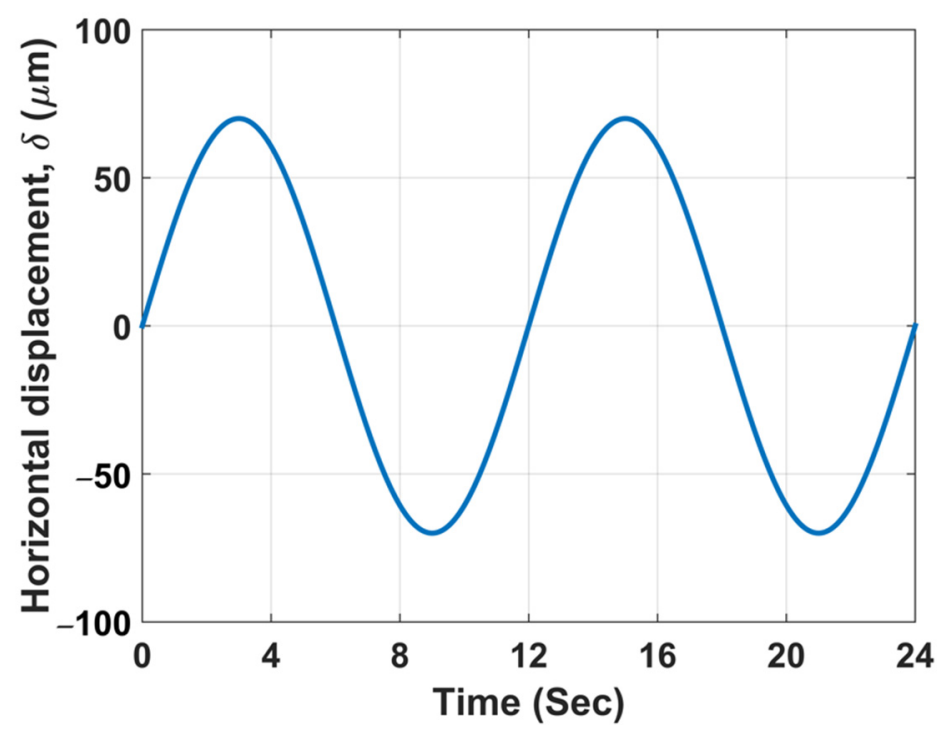

Figure 6. Horizontal input displacement cycle supplied to the piezoelectric motor to move the mica disk. 


\section{Results and Discussion}

\subsection{In Situ Visualization of Contact Interface}

In order to investigate the motion of micrometer spheres between two flat surfaces, an approach cycle was performed using the elevation stage to raise the mica disk that carries the microspheres, bring it in contact with the fixed upper surface, and then move the mica disk horizontally using the piezoelectric actuator. The approach and sliding cycles were executed in room environment and dry condition. It is crucial that minimal normal force is applied on the steel microspheres to avoid damaging them. The cantilever beam setup (with a stiffness of $2.1 \mathrm{mN} / \mu \mathrm{m}$ ), along with the extremely low vertical speed $(0.1 \mu \mathrm{m} / \mathrm{s})$ used in this instrument, ensures that only a small normal load is exerted on the microspheres. Throughout the approach cycle, the proximity sensor measures the vertical displacement of the mica disk. Therefore, contact initiation is detected when the proximity sensor reads no movement of the mica disk as the elevation stage moves upward.

Moreover, the optical microscope was used to confirm the contact initiation by visualizing the microspheres. Figure 7 depicts the micrographs of a steel microsphere in between the mica disk and the sapphire window. From Figure 7b, the presence of Newton's rings pattern around the contact area (the region in the center) corroborates the surfaces' contact. It should be noted that tens of microspheres (with diameters in the range of 41 to $48 \mu \mathrm{m}$ ) were placed on the mica disk. Therefore, it is expected that the contact will occur between multiple microspheres and the upper sapphire window. However, the optical microscope was focused on a single microsphere during the micro-sliding test to carefully study the movement of the spherical particle between flat surfaces. This finding clearly indicates that the microspheres prevented direct contact between the mica and the sapphire surfaces.

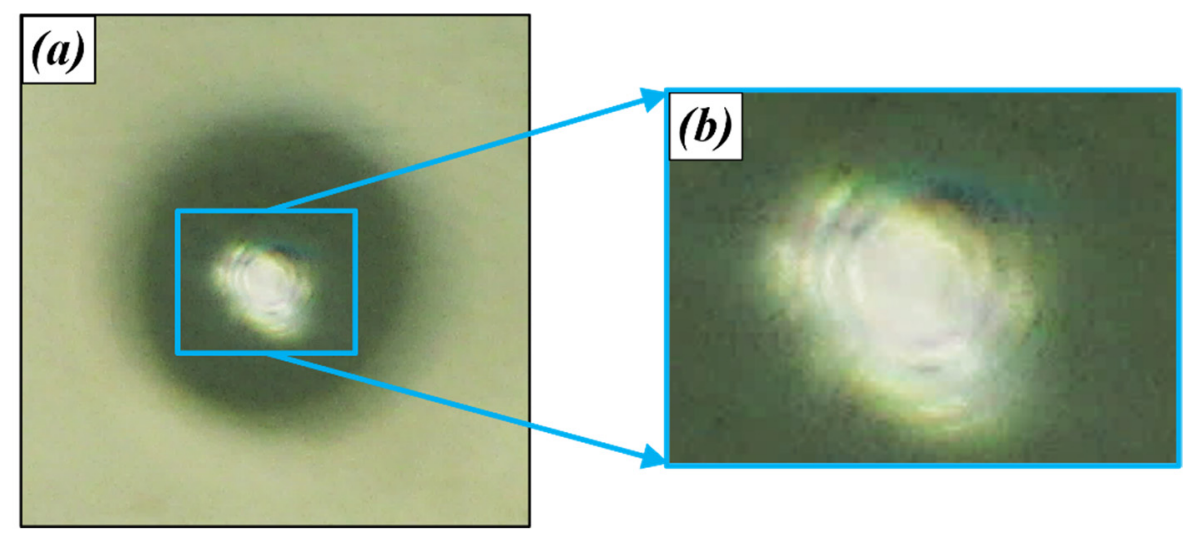

Figure 7. (a) Micrograph of a steel microsphere in between the mica disk and the sapphire window illustrating (b) the presence of Newton's rings pattern around the contact area (the region in the center).

This experimental setup allowed us to study the lubrication mechanism of solid microand nano-particles between flat surfaces. During boundary and mixed lubrication, the gap between sliding surfaces is very small. Therefore, when a lubricant with solid additives is used, those solid particles will bear some of the applied load, prevent contact between flat surfaces, and, as a result, reduce friction and wear.

\subsection{Micro-Sliding Investigation}

Contact between steel microspheres and the upper sapphire window was initiated. The piezoelectric motor was operated to slide the mica disk with microspheres parallel to the sapphire surface using a sinusoidal cycle (the amplitude of $70 \mu \mathrm{m}$ ). Hence, the total horizontal displacement of the mica disk was $140 \mu \mathrm{m}$. At the same time, the optical microscope located directly above the sapphire window was utilized to observe the motion of the microspheres. However, throughout the micro-sliding test, the optical microscope was focused on a single microsphere to scrutinize its movement between the flat surfaces. 
Moreover, the micro-sliding investigation was conducted at a very slow operational frequency $(0.08 \mathrm{~Hz})$ to closely explore the motion of steel microspheres between the two flat surfaces. The digital camera was used to video record the motion of the microsphere during the micro-sliding test. Later, the in situ acquired video (refer to Supplementary File S1) was processed to extract digital micrographs of the surfaces' interface throughout the micro-sliding cycle (see Figure 8). The total traveling distance of the microsphere was measured by tracing the center of the microsphere, while the total travel distance of the mica disk was measured by tracing a mark on the mica disk. Figure $8 \mathrm{~d}$ illustrates the position of the steel microsphere in the extracted digital images (Figure 8a-c) relative to the input displacement cycle given to the piezoelectric motor. It can be seen from Figure 8 that the total traveling distance of the microsphere was $70 \mu \mathrm{m}$ while the total traveling distance of the mica disk was $140 \mu \mathrm{m}$. Thus, the total lateral movement of the mica disk $(140 \mu \mathrm{m})$ was twice the displacement of the steel microsphere $(70 \mu \mathrm{m})$.
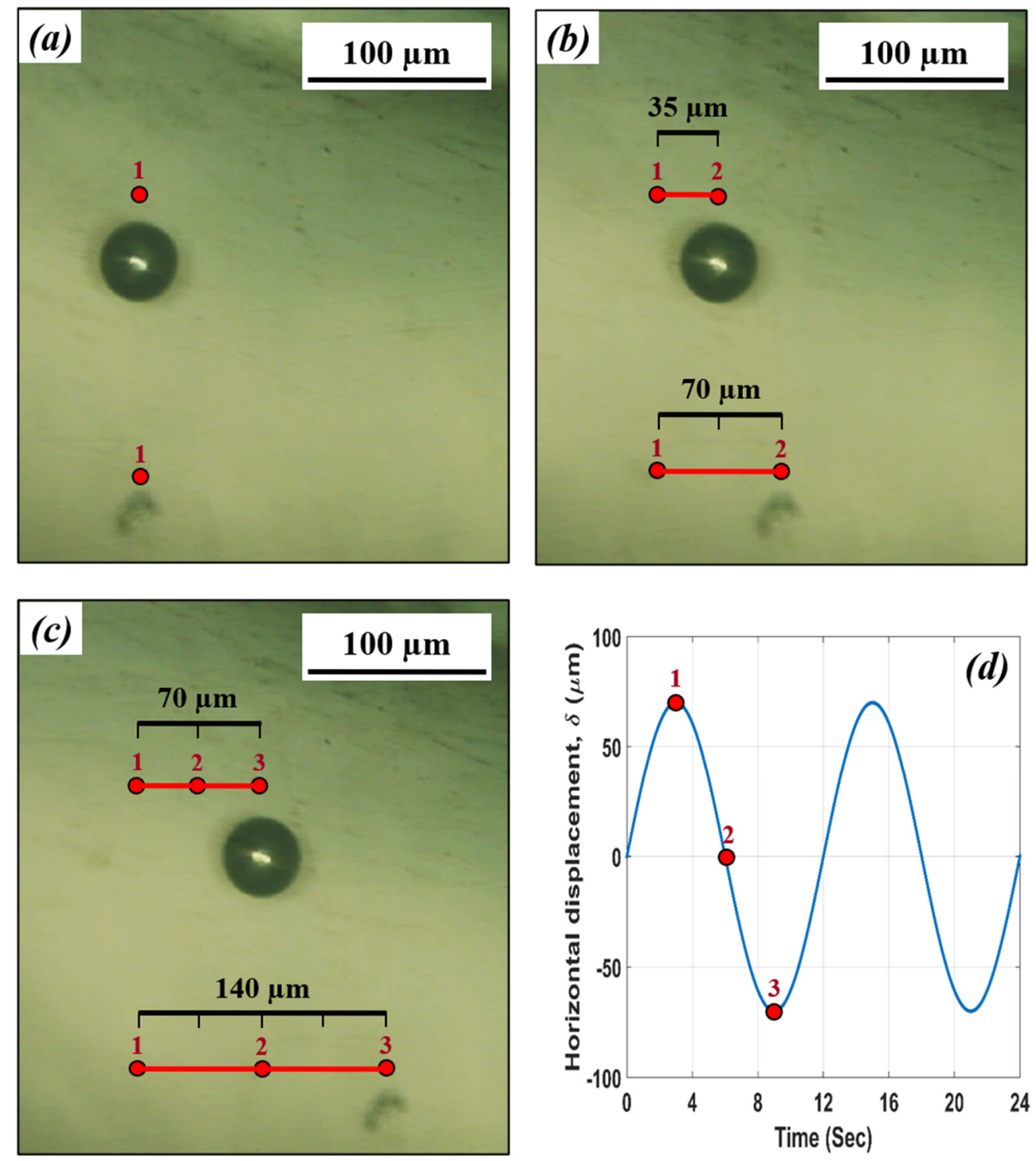

Figure 8. Digital micrographs of a stainless-steel microsphere at (a) initial, (b) middle, and (c) end of the lateral motion cycle of the mica disk. (d) Position of the microsphere in micrographs relative to displacement cycle given to the piezoelectric motor. 


\subsection{Analysis of the Motion of the Microsphere between Flat Surfaces}

The motion of a sphere between two flat surfaces can be pure slipping, pure rolling, or rolling with slipping. To determine the type of motion, it is required to measure the traveled distance of the sphere and flat surfaces.

It is well known that in a slipping (pure translational) motion, every point of the rigid sphere will have the same velocity and in the same direction. On the other hand, rolling motion is a combination of translation motion and rotational motion. Therefore, when a sphere is rolling on a flat surface without slipping, the point of contact of the sphere with the fixed flat surface will have zero velocity.

Figure 9 depicts a schematic of the velocity at different points on a microsphere between two flat surfaces. If the bottom point of the microsphere was moved with a velocity $V_{1}$, then we have three possible scenarios. In the first scenario, the center of the microsphere has the same velocity as $V_{1}$, which means that the microsphere is in slipping (pure translational) motion. In the second scenario, the center of the microsphere has a velocity of $\frac{1}{2} V_{1}$, which means that the microsphere is in rolling without slipping motion. In the third scenario, the center of the sphere has a velocity between $\frac{1}{2} V_{1}$ and $V_{1}$, which means that the microsphere is in rolling with slipping motion.

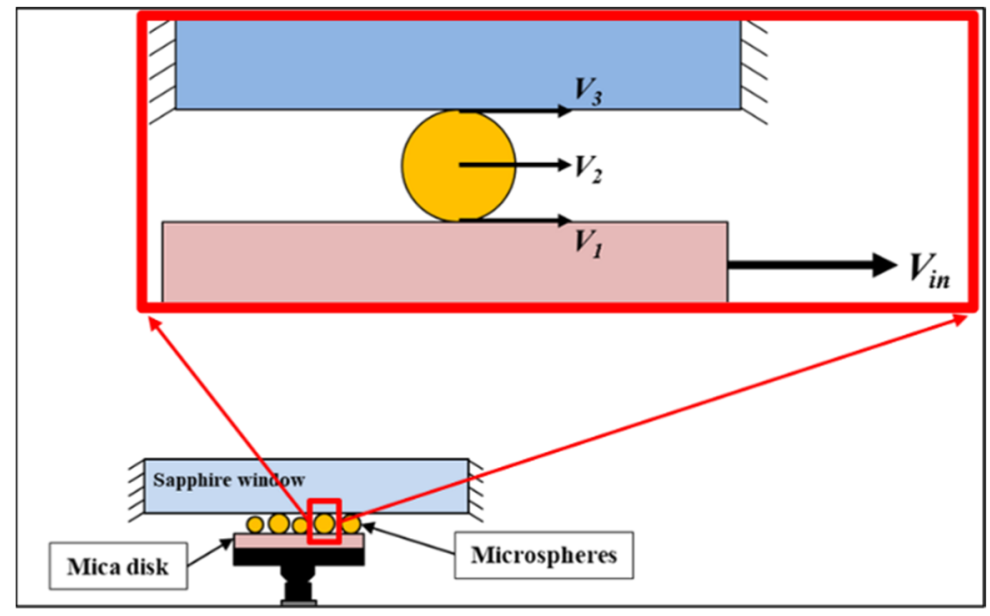

Figure 9. Schematic of a microsphere between the two flat surfaces where $V_{1}, V_{2}, V_{3}$ are velocities of the sphere at the bottom, center, and top point, respectively.

In this investigation, the piezoelectric motor was utilized to move the mica surface (beneath microspheres) horizontally against the stationary sapphire window (above the microspheres). The upper flat surface was stationary (i.e., $V_{3}=0$ ) and the total horizontal displacement of the lower flat disk was $140 \mu \mathrm{m}$ in $6 \mathrm{~s}$ (i.e., $V_{1}=23.33 \mu \mathrm{m} / \mathrm{s}$ ). As shown in Figure 8 , the overall lateral displacement of the steel microsphere was half $(70 \mu \mathrm{m})$ the displacement of the mica disk $(140 \mu \mathrm{m})$. It should be noted that the total lateral displacement of the steel microsphere was $70 \mu \mathrm{m}$ in $6 \mathrm{~s}$ (i.e., $V_{2}=11.67 \mu \mathrm{m} / \mathrm{s}$ ). Hence, the velocity of the center of the microsphere was half the velocity of the mica disk. These results demonstrate that the motion of the microsphere between two flat surfaces was rolling without slipping (pure rolling motion). This rolling without slipping motion of micro-sized spherical particles placed in-between two flat bodies gives support to the idea of adding rigid spherical particles to liquid lubricants to further minimize frictional and wear losses by acting as micro and nanoscale ball bearings.

During the micro-sliding experiments, an in-line load sensor was employed to measure the friction force experienced by the microspheres (see Figure 1). Figure 10 depicts the friction force measurements versus time at different applied normal loads ( 7 and $14 \mathrm{mN}$ ). It should be noted that a relatively small sliding distance was used in the friction experiments to visually examine the motion of the solid particles and measure the friction force. Longer sliding distance experiments will result in losing the focus of the optical microscope 
on the solid particles. It is clear from Figure 10 that very low friction force was measured, and the obtained coefficient of friction was about 0.03 . It is well known that rolling friction is considerably lower than sliding friction, and the rolling coefficient of friction is less than $0.1[40,41]$. Therefore, the low coefficient of friction obtained from the micro-sliding experiments confirms the rolling motion of the microspheres between the mating surfaces.

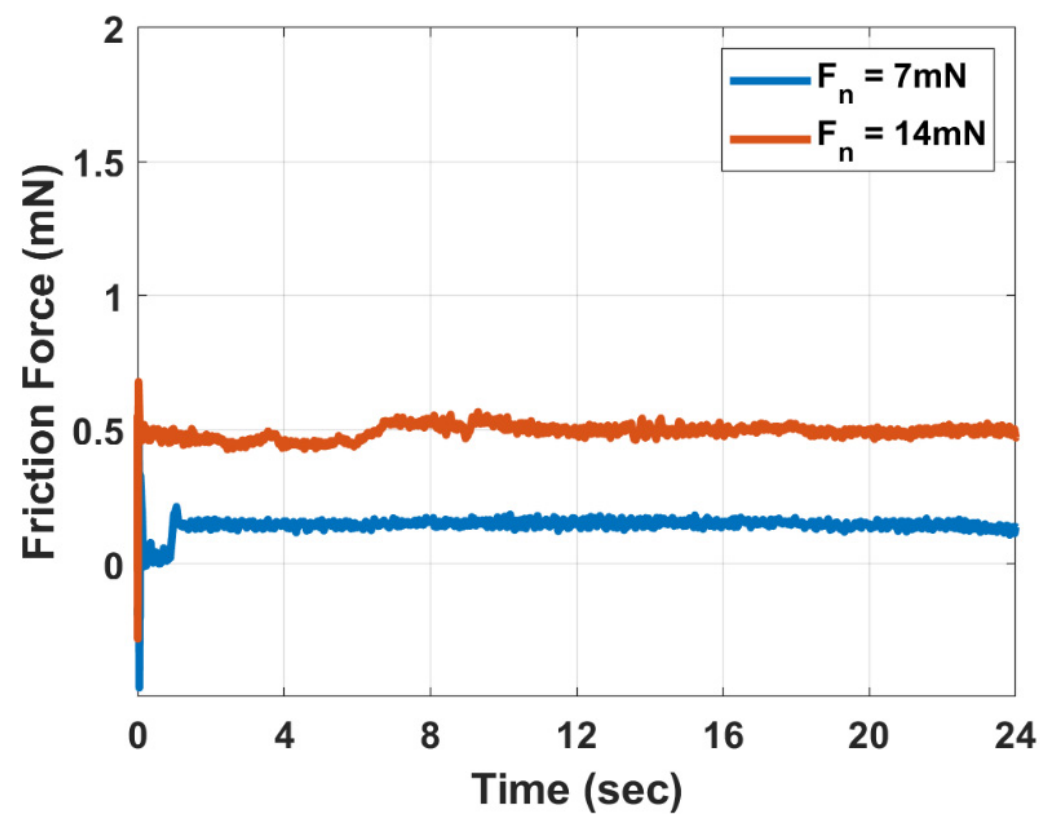

Figure 10. Friction force versus time at different applied normal loads.

The major purpose of the current work was to verify the hypothesis that micro- and nano-spherical particles could introduce the rolling motion within the contacting surface and, as a result, reduce friction and wear. Therefore, relatively large particles $(\sim 50 \mu \mathrm{m})$ were used to visualize their rolling motion between mating surfaces. However, different sizes and types of micro- and nano-particles are available and can be selected based on the condition and roughness of the mating surfaces.

\section{Conclusions}

An optical micro-sliding apparatus was developed to examine the movement of micron-sized spherical particles placed between flat bodies to better understand their lubrication mechanism when used as oil additives. The experimental instrument performed velocity and friction force measurements during the micro-sliding experiments. The following points can be concluded from our study:

(1) The spherical particles had a diameter ranging from 41 to $48 \mu \mathrm{m}$ and this is useful to accommodate for the irregularities caused by the surface roughness and/or condition of mating surfaces.

(2) In micro-sliding experiments, it was shown that the velocity of the center of the steel microsphere was half the velocity of the lower disk, and friction force measurements revealed a very low coefficient of friction (about 0.03), confirming the pure rolling motion of the microspheres.

(3) The key outcome of this study was the verification of the assumption that spherical particles can be used to prevent direct contact and introduce rolling motion between mating surfaces.

(4) This outcome supports the idea that micro- and nano-sized spherical particles can be utilized as ball bearings at the microscale to improve the tribological behavior of current lubricant oils. 
Supplementary Materials: The following are available online at https:/ / www.mdpi.com/article/ $10.3390 /$ lubricants $9080081 /$ s1, Video S1: Video record of the motion of a microsphere during the micro-sliding test.

Funding: This research received no external funding.

Institutional Review Board Statement: Not applicable.

Informed Consent Statement: Not applicable.

Data Availability Statement: Not applicable.

Acknowledgments: The author gratefully acknowledges the support of the Kuwait University General Facility (Grant No. GE 01/07). Electron microscopy studies were conducted at KUNRF. The author also acknowledges Farshid Sadeghi (Purdue University, Mechanical Engineering Tribology Laboratory) for his support in using his laboratory to conduct the experimental work using the optical micro-sliding instrument.

Conflicts of Interest: The author declares no conflict of interest.

\section{References}

1. Hamrock, B.J.; Schmid, S.R.; Jacobson, B.O. Fundamentals of Fluid Film Lubrication; CRC Press: Boca Raton, FL, USA, 2004.

2. Sun, J.; Du, S. Application of graphene derivatives and their nanocomposites in tribology and lubrication: A review. RSC Adv. 2019, 9, 40642-40661. [CrossRef]

3. Shi, S.C.; Jiang, S.Z. Influence of graphene/copper hybrid nanoparticle additives on tribological properties of solid cellulose lubricants. Surf. Coat. Technol. 2020, 389, 125655. [CrossRef]

4. He, G.; Li, Y.; Wu, L.; Wang, Y.; Liu, M.; Yuan, J.; Men, X. Synergy of core-shell Cu@ rGO hybrids for significantly improved thermal and tribological properties of polyimide composites. Tribol. Int. 2021, 161, 107091. [CrossRef]

5. Kalin, M.; Kogovšek, J.; Remskar, M. Mechanisms and improvements in the friction and wear behavior using MoS $_{2}$ nanotubes as potential oil additives. Wear 2012, 280-281, 36-45. [CrossRef]

6. Wu, Y.; Tsui, W.; Liu, T. Experimental analysis of tribological properties of lubricating oils with nanoparticle additives. Wear 2007, 262, 819-825. [CrossRef]

7. Tang, Z.; Li, S. A review of recent developments of friction modifiers for liquid lubricants (2007-present). Curr. Opin. Solid State Mater. Sci. 2014, 18, 119-139. [CrossRef]

8. Singh, Y.; Sharma, A.; Singh, N.; Singla, A. Effect of alumina nanoparticles as additive on the friction and wear behavior of polanga-based lubricant. SN Appl. Sci. 2019, 1, 281. [CrossRef]

9. Martin, J.M.; Ohmae, N. Nanolubricants; John Wiley \& Sons: Hoboken, NJ, USA, 2008; Volume 13.

10. Manu, B.R.; Gupta, A.; Jayatissa, A.H. Tribological Properties of 2D Materials and Composites-A Review of Recent Advances. Materials 2021, 14, 1630. [CrossRef]

11. Alazemi, A.A.; Dysart, A.D.; Pol, V.G. Experimental Investigation of the Mechanical and Surface Properties of Sub-Micron Carbon Spheres. Lubricants 2020, 8, 77. [CrossRef]

12. Ghaednia, H.; Jackson, R.L. The Effect of Nanoparticles on the Real Area of Contact, Friction, and Wear. J. Tribol. 2013, 135, 041603. [CrossRef]

13. Hu, C.; Bai, M.; Lv, J.; Kou, Z.; Li, X. Molecular dynamics simulation on the tribology properties of two hard nanoparticles (diamond and silicon dioxide) confined by two iron blocks. Tribol. Int. 2015, 90, 297-305. [CrossRef]

14. Sgroi, M.; Gili, F.; Mangherini, D.; Lahouij, I.; Dassenoy, F.; Garcia, I.; Odriozola, I.; Kraft, G. Friction reduction benefits in valve-train system using IF-MoS 2 added engine oil. Tribol. Trans. 2015, 58, 207-214. [CrossRef]

15. Gullac, B.; Akalin, O. Frictional Characteristics of IF-WS 2 Nanoparticles in Simulated Engine Conditions. Tribol. Trans. 2010, 53, 939-947. [CrossRef]

16. Tannous, J.; Dassenoy, F.; Lahouij, I.; Le Mogne, T.; Vacher, B.; Bruhács, A.; Tremel, W. Understanding the tribochemical mechanisms of IF-MoS 2 nanoparticles under boundary lubrication. Tribol. Lett. 2011, 41, 55-64. [CrossRef]

17. Alazemi, A.A.; Etacheri, V.; Dysart, A.D.; Stacke, L.-E.; Pol, V.G.; Sadeghi, F. Ultrasmooth Submicrometer Carbon Spheres as Lubricant Additives for Friction and Wear Reduction. ACS Appl. Mater. Interfaces 2015, 7, 5514-5521. [CrossRef]

18. Alazemi, A.A.; Dysart, A.D.; Phuah, X.L.; Pol, V.G.; Sadeghi, F. MoS $_{2}$ nanolayer coated carbon spheres as an oil additive for enhanced tribological performance. Carbon 2016, 110, 367-377. [CrossRef]

19. Alazemi, A.A. Experimental Investigation of Friction Fundamentals at the Microscale. Ph.D. Thesis, Purdue University, West Lafayette, IN, USA, 2017.

20. Rapoport, L.; Feldman, Y.; Homyonfer, M.; Cohen, H.; Sloan, J.; Hutchison, J.; Tenne, R. Inorganic fullerene-like material as additives to lubricants: Structure-function relationship. Wear 1999, 225-229, 975-982. [CrossRef]

21. Rapoport, L.P.; Bilik, Y.; Feldman, Y.A.; Homyonfer, M.; Cohen, S.; Tenne, R. Hollow nanoparticles of $\mathrm{WS}_{2}$ as potential solid-state lubricants. Nature 1997, 387, 791-793. [CrossRef] 
22. Teoh, Y.; How, H.; Sher, F.; Le, T.; Nguyen, H.; Yaqoob, H. Fuel Injection Responses and Particulate Emissions of a CRDI Engine Fueled with Cocos nucifera Biodiesel. Sustainability 2021, 13, 4930. [CrossRef]

23. Habibullah, M.; Masjuki, H.; Kalam, A.; Zulkifli, N.; Masum, B.; Arslan, A.; Gulzar, M. Friction and wear characteristics of Calophyllum inophyllum biodiesel. Ind. Crop. Prod. 2015, 76, 188-197. [CrossRef]

24. Yaqoob, H.; Teoh, Y.H.; Jamil, M.A.; Rasheed, T.; Sher, F. An Experimental Investigation on Tribological Behaviour of Tire-Derived Pyrolysis Oil Blended with Biodiesel Fuel. Sustainability 2020, 12, 9975. [CrossRef]

25. Teoh, Y.H.; How, H.G.; Sher, F.; Le, T.D.; Ong, H.C.; Nguyen, H.T.; Yaqoob, H. Optimization of Fuel Injection Parameters of Moringa oleifera Biodiesel-Diesel Blend for Engine-Out-Responses Improvements. Symmetry 2021, 13, 982. [CrossRef]

26. Mujtaba, M.A.; Cho, H.M.; Masjuki, H.H.; Kalam, M.A.; Farooq, M.; Soudagar, M.E.M.; Gul, M.; Ahmed, W.; Afzal, A.; Bashir, S.; et al. Effect of alcoholic and nano-particles additives on tribological properties of diesel-palm-sesame-biodiesel blends. Energy Rep. 2021, 7, 1162-1171. [CrossRef]

27. Fayaz, H.; Mujtaba, M.; Soudagar, M.E.M.; Razzaq, L.; Nawaz, S.; Nawaz, M.A.; Farooq, M.; Afzal, A.; Ahmed, W.; Khan, T.Y.; et al. Collective effect of ternary nano fuel blends on the diesel engine performance and emissions characteristics. Fuel 2021, 293, 120420. [CrossRef]

28. Nikolakopoulos, P.G.; Mavroudis, S.; Zavos, A. Lubrication performance of engine commercial oils with different performance levels: The effect of engine synthetic oil aging on piston ring tribology under real engine conditions. Lubricants $2018,6,90$. [CrossRef]

29. Lin, Q.; Bao, Q.; Li, K.; Khonsari, M.; Zhao, H. An investigation into the transient behavior of journal bearing with surface texture based on fluid-structure interaction approach. Tribol. Int. 2018, 118, 246-255. [CrossRef]

30. Xie, Z.; Zhang, Y.; Zhou, J.; Zhu, W. Theoretical and experimental research on the micro interface lubrication regime of water lubricated bearing. Mech. Syst. Signal Process. 2021, 151, 107422. [CrossRef]

31. Xie, Z.; Zhu, W. An investigation on the lubrication characteristics of floating ring bearing with consideration of multi-coupling factors. Mech. Syst. Signal Process. 2022, 162, 108086. [CrossRef]

32. Lin, Q.; Wei, Z.; Wang, N.; Chen, W. Analysis on the lubrication performances of journal bearing system using computational fluid dynamics and fluid-structure interaction considering thermal influence and cavitation. Tribol. Int. 2013, 64, 8-15. [CrossRef]

33. Zhang, H.; Hua, M.; Dong, G.-N.; Zhang, D.-Y.; Chin, K.-S. Boundary slip surface design for high speed water lubricated journal bearings. Tribol. Int. 2014, 79, 32-41. [CrossRef]

34. Hu, C.; Bai, M.; Lv, J.; Wang, P.; Li, X. Molecular dynamics simulation on the friction properties of nanofluids confined by idealized surfaces. Tribol. Int. 2014, 78, 152-159. [CrossRef]

35. Capozza, R.; Fasolino, A.; Ferrario, M.; Vanossi, A. Lubricated friction on nanopatterned surfaces via molecular dynamics simulations. Phys. Rev. B 2008, 77, 235432. [CrossRef]

36. Li, S.; Ren, L.; Bai, Z. Friction performance and mechanisms of calcined products of $\mathrm{Mg} / \mathrm{Al}$ layered double hydroxides as lubricant additives. Appl. Surf. Sci. 2019, 470, 979-990. [CrossRef]

37. Alazemi, A.A.; Sadeghi, F.; Stacke, L.-E. Adhesion and Friction Force Measurements Using an Optical Micro-Apparatus. Tribol. Lett. 2016, 64, 49. [CrossRef]

38. Pacquentin, W.; Caron, N.; Oltra, R. Effect of microstructure and chemical composition on localized corrosion resistance of a AISI 304L stainless steel after nanopulsed-laser surface melting. Appl. Surf. Sci. 2015, 356, 561-573. [CrossRef]

39. Alazemi, A.A.; Ghosh, A.; Sadeghi, F.; Stacke, L.-E. Experimental Investigation of the Correlation between Adhesion and Friction Forces. Tribol. Lett. 2016, 62, 1-12. [CrossRef]

40. Bhushan, B. Introduction to Tribology; John Wiley \& Sons: Hoboken, NJ, USA, 2013; pp. 199-266.

41. Gupta, B.S. Friction in Textile Materials; Elsevier: Amsterdam, The Netherlands, 2008; pp. 37-66. 